\title{
ABELIAN AND TAUBERIAN THEOREMS FOR 0-REGULARLY VARYING FUNCTIONS
}

\author{
J. L. GELUK
}

ABSTRACT. A general kernel Abelian and Tauberian theorem is proved for functions $f$ satisfying $\varlimsup_{t \rightarrow \infty} f(t x) / f(t)<\infty$ for $x>0$.

Introduction. In this paper we prove Abelian and Tauberian theorems for integral transforms for two classes of functions. First we give the definition of the classes of functions we consider.

Definition (SEE [1]). A measurable function $f: \mathbf{R}^{+} \rightarrow \mathbf{R}^{+}$is 0-regularly varying (notation $f \in \mathrm{RO}$ ) if one of the following equivalent conditions holds:

(i)

$$
\Phi_{f}(x):=\varlimsup_{t \rightarrow \infty} \frac{f(t x)}{f(t)}<\infty \text { for } x>0 .
$$

(ii) There exist constants $c_{1}, c_{2}, t_{0} \geqslant 0$ and $\alpha, \beta \in \mathbf{R}$ such that for $t>t_{0}$ and $x \geqslant 1$

$$
c_{1} x^{\alpha} \leqslant \frac{f(t x)}{f(t)} \leqslant c_{2} x^{\beta}
$$

(iii)

$$
\lim _{x \rightarrow \infty} \frac{\log \varlimsup_{t \rightarrow \infty} f(t x) / f(t)}{\log x}<\infty
$$

and

$$
\lim _{x \rightarrow \infty} \frac{\log \lim _{t \rightarrow \infty} f(t x) / f(t)}{\log x}>-\infty .
$$

(iv) There exists a positive nondecreasing function $\rho$, a positive nonincreasing function $\psi$ and constants $\alpha, \beta \in \mathbf{R}$ such that

$$
f(t) \asymp t^{\alpha} \rho(t) \asymp t^{\beta} \psi(t) \quad(t \rightarrow \infty) .
$$

(We use the symbol $a(t) \asymp b(t)(t \rightarrow \infty)$ to mean $a(t), b(t)>0$ and $\log a(t) / b(t)$ is bounded for $t>\infty$.) 
The left-hand side of (3) is called the upper index of $f(\overline{\operatorname{index}} f)$ and the left-hand side of (4) is called the lower index of $f$ (index $f$ ). If $f \in \mathrm{RO}$ we can take any $\alpha<$ index $f$ and $\beta>\overline{\operatorname{index}} f$ in (2) and (5). For a review of properties of 0 -regularly varying functions the reader is referred to [2].

Definition. A nondecreasing function $f: \mathbf{R}^{+} \rightarrow \mathbf{R}$ is asymptotically balanced if there exists a constant $x>1$ and a function $a$ such that

$$
f(t x)-f(t) \asymp a(t) \quad(t \rightarrow \infty) .
$$

It is well known that (6) implies $a \in \mathrm{RO}$ (see [5]).

We study integral transforms of the above classes of functions and use the following notation:

$$
\hat{f}(\sigma)=-\sigma \int_{0}^{\infty} \rho^{\prime}(\sigma s) f(s) d s, \quad \sigma>0 .
$$

In this paper the function $\rho:[0, \infty) \rightarrow[0, \infty)$ is differentiable with $\rho^{\prime}(x) \leqslant 0$ for $x>0, \rho(\infty)=0$ and $\rho(x)>0$ for some $x>0$. The integral in (7) is supposed to be finite for $\sigma>0$.

In case the limit in (1) exists it is possible to deduce a Tauberian theorem using Wiener's approach. This is shown in a paper by Bingham and Teugels [3]. A Tauberian theorem concerning functions $f$ satisfying (6) with $\asymp$ replaced by $~$, where the function $a$ is slowly varying, is proved in [4]. For the case of the Laplace-Stieltjes transform a Tauberian theorem for nondecreasing 0-regularly varying functions is given in de Haan and Stadtmüller [6].

Results. In the following, $c_{i}(i=1,2, \ldots)$ denotes a positive finite constant not necessarily the same at each appearance.

First we give the following Abelian result.

THeOREM 1. Suppose $f \in \mathrm{RO}$ is locally bounded, $\alpha:=\operatorname{index} f, \beta:=\overline{\operatorname{index}} f$,

$$
-\int_{0}^{1} s^{\alpha-\delta} \rho^{\prime}(s) d s<\infty, \quad-\int_{1}^{\infty} s^{\beta+\delta} \rho^{\prime}(s) d s<\infty
$$

for some $\delta>0$.

The above assumptions imply

$$
f(t) \asymp \hat{f}(1 / t) \quad(t \rightarrow \infty),
$$

hence

$$
\varlimsup_{t \rightarrow \infty} \frac{\hat{f}(1 / t x)}{\hat{f}(1 / t)}<\infty \quad \text { for } x>0 .
$$

In order to prove this result we need the following.

Lemma. Suppose $0<\operatorname{index} f \leqslant \overline{\operatorname{index}} f<\infty$ and $f$ is locally bounded on $\mathbf{R}^{+}$. Then there exist $t_{0}$ and $c_{1}$ such that for $t \geqslant t_{0}$ and $0<x \leqslant 1$

$$
f(t x) / f(t) \leqslant c_{1} \text {. }
$$

Suppose $\alpha:=\operatorname{index} f \leqslant 0, \overline{\operatorname{index}} f<\infty$ and $f$ is locally bounded on $\mathbf{R}^{+}$. For $\delta>0$ arbitrary, there exist $t_{1}$ and $c_{2}$ such that for $t \geqslant t_{1}$ and $0<x \leqslant 1$

$$
f(t x) / f(t) \leqslant c_{2} x^{\alpha-\delta} .
$$


Proof. The proof of this Lemma is given by application of the first inequality in (2) and applying the first result to the function $t^{-\alpha+\delta} f(t)$.

Proof of Theorem 1. Take $\delta>0$ such that (8) holds true. Then using (11) and (2) we find

$$
\begin{aligned}
\frac{\hat{f}(1 / t)}{f(t)} & =-\int_{0}^{1} \rho^{\prime}(s) \frac{(t s)^{-\alpha+\delta} f(t s)}{t^{-\alpha+\delta} f(t)} s^{\alpha-\delta} d s-\int_{1}^{\infty} \rho^{\prime}(s) \frac{f(t s)}{f(t)} d s \\
& \leqslant-c_{1} \int_{0}^{1} \rho^{\prime}(s) s^{\alpha-\delta} d s-c_{2} \int_{1}^{\infty} \rho^{\prime}(s) s^{\beta+\delta} d s .
\end{aligned}
$$

Take a nondecreasing function $\phi$ on $\left(t_{0}, \infty\right)$ such that $f(t) \asymp t^{\alpha-\delta} \phi(t)$ for $t>t_{0}$. Then for $t>t_{0}$

$$
\begin{aligned}
\hat{f}\left(\frac{1}{t}\right) & \geqslant-\int_{1}^{\infty} \rho^{\prime}(s) f(t s) d s \geqslant-c_{3} t^{\alpha-\delta} \int_{1}^{\infty} \rho^{\prime}(s) s^{\alpha-\delta} \phi(s t) d s \\
& \geqslant-c_{3} t^{\alpha-\delta} \phi(t) \int_{1}^{\infty} \rho^{\prime}(s) s^{\alpha-\delta} d s \geqslant c_{4} f(t) .
\end{aligned}
$$

Now (1), (13) and (14) imply (9) and (10).

Next we prove a Tauberian converse of Theorem 1 . Theorem 1 concerns functions $f$ tending to infinity as well as functions tending to zero. The following two theorems give converse statements for functions $f$ bounded away from zero (Theorem 2) and infinity (Theorem 3).

THEOREM 2. Suppose $f:[0, \infty) \rightarrow[0, \infty)$ is of bounded variation on finite intervals, and satisfies

$$
\begin{gathered}
f(0+)=0, \\
\lim _{x \rightarrow \infty} f(x)>0 \text { and } \varlimsup_{x \rightarrow \infty} f(x)=\infty,
\end{gathered}
$$

and, for $\lambda>1$ fixed,

$$
\frac{\lim }{t \rightarrow \infty} \inf _{t \leqslant t^{\prime} \leqslant \lambda t}\left\{f\left(t^{\prime}\right)-f(t)\right\}>-\infty
$$

Suppose $\rho$ satisfies

$$
\sum_{k=1}^{\infty} k\left\{\rho\left(\lambda^{-k-1}\right)-\rho\left(\lambda^{-k}\right)\right\}<\infty \text { and } \sum_{k=1}^{\infty} \rho\left(\lambda^{k}\right)<\infty .
$$

Let

$$
\Phi_{\hat{f}}(x):=\varlimsup_{t \rightarrow \infty} \frac{\hat{f}(1 / t x)}{\hat{f}(1 / t)}<\infty \text { for } x>1 .
$$

Then $a=\overline{\operatorname{index}} \hat{f}(1 / t)$ exists.

Suppose

$$
-\int_{1}^{\infty} t^{\alpha} \rho^{\prime}(t) d t<\infty \quad \text { for some } \alpha>a
$$


Then the above assumptions imply (9), and hence,

$$
\lim _{t \rightarrow \infty} \frac{f(t x)}{f(t)}<\infty \text { for } x>1
$$

Proof. Without loss of generality we may suppose $\rho(1)>0$. For $T=1 / \sigma>0$ we have

$$
\hat{f}(\sigma)=\rho(1) f(T)-\int_{0}^{T} \sigma \rho^{\prime}(\sigma t) f(t) d t+\int_{T}^{\infty} \rho(\sigma t) d f(t) .
$$

Using (17) we have

$$
\begin{aligned}
\int_{T}^{\infty} \rho(\sigma t) d f(t) & =\sum_{k=0}^{\infty} \int_{T \lambda^{k}}^{T \lambda^{k+1}} \rho(\sigma t) d f(t) \\
& \geqslant \sum_{k=0}^{\infty} \rho\left(\lambda^{k}\right) \inf \left\{f(\xi)-f\left(T \lambda^{k}\right)\right\} \\
& \geqslant-c \sum_{k=0}^{\infty} \rho\left(\lambda^{k}\right):=-c_{1},
\end{aligned}
$$

where the infinum is taken over $\xi \in\left[T \lambda^{k}, T \lambda^{k+1}\right]$. This proves

$$
\hat{f}(\sigma) \geqslant \rho(1) f(1 / \sigma)-c_{1} \quad(\sigma>0) .
$$

For $\sigma>0$ we write

$$
\hat{f}(\sigma)=-\int_{0}^{\beta} f(t T) \rho^{\prime}(t) d t-\int_{\beta}^{\infty} f(t T) \rho^{\prime}(t) d t,
$$

where $\beta>0$ is specified later.

Using (17) for $t>0$ there exists $t_{0} \in[t, \lambda t]$ such that

$$
\begin{aligned}
\inf _{t \leqslant t^{\prime} \leqslant \lambda t}\left\{f(\lambda t)-f\left(t^{\prime}\right)\right\} & \geqslant f(\lambda t)-f\left(t_{0}\right)-1 \\
& \geqslant \inf _{t_{0} \leqslant t^{\prime} \leqslant \lambda t_{0}}\left\{f\left(t^{\prime}\right)-f\left(t_{0}\right)\right\}-1 \geqslant-c
\end{aligned}
$$

since $t \leqslant t_{0} \leqslant \lambda t \leqslant \lambda t_{0}$. Since $\rho$ is nonincreasing we have

$$
\begin{aligned}
-\int_{0}^{\beta} f(t T) \rho^{\prime}(t) d t & =-\sum_{k=0}^{\infty} \int_{\beta \lambda^{-k-1}}^{\beta \lambda^{-k}} f(t T) \rho^{\prime}(t) d t \\
& \leqslant-\sum_{k=0}^{\infty} \sup _{\beta \lambda^{-k-1} T \leqslant \xi \leqslant \beta \lambda^{-k} T} f(\xi)\left\{\rho\left(\beta \lambda^{-k}\right)-\rho\left(\beta \lambda^{-k-1}\right)\right\} \\
& \leqslant \sum_{k=0}^{\infty}\left[f\left(\beta \lambda^{-k} T\right)+c\right]\left\{\rho\left(\beta \lambda^{-k-1}\right)-\rho\left(\beta \lambda^{-k}\right)\right\},
\end{aligned}
$$

the last inequality being justified by (24).

Repeated application of (17) gives, for $k=1,2, \ldots$,

$$
f\left(\beta \lambda^{-k} T\right) \leqslant f(\beta T)+k c .
$$

Hence

$$
-\int_{0}^{\beta} f(t T) \rho^{\prime}(t) d t \leqslant c_{2} f(\beta T)+c_{3},
$$


where $c_{2}=\rho(0)-\rho(\beta)$ and $c_{3}=c \sum_{k=0}^{\infty}(k+1)\left\{\rho\left(\beta \lambda^{-k-1}\right)-\rho\left(\beta \lambda^{-k}\right)\right\}$. In order to estimate the second integral in (23) we note that by (2) there exist constants $c_{4}$ and $\sigma_{0}$ such that for $t \geqslant 1$ and $\sigma<\sigma_{0}$

$$
\hat{f}(\sigma / t) \leqslant c_{4} t^{\alpha} \hat{f}(\sigma) .
$$

Using the above inequality and (22) we have for $\sigma<\sigma_{0}$ and $\beta \geqslant 1$

$$
\begin{aligned}
-\int_{\beta}^{\infty} f(t T) \rho^{\prime}(t) d t & \leqslant-\rho(1)^{-1} \int_{\beta}^{\infty}\left\{\hat{f}\left(\frac{\sigma}{t}\right)+c_{1}\right\} \rho^{\prime}(t) d t \\
& \leqslant c_{5} \hat{f}(\sigma)+c_{6},
\end{aligned}
$$

where $c_{5}=-c_{4} \rho(1)^{-1} \int_{\beta}^{\infty} t^{\alpha} \rho^{\prime}(t) d t<\infty$ and $c_{6}=c_{1} \rho(1)^{-1} \rho(\beta)$. Combination of (23), (25) and (26) gives for $\sigma<\sigma_{0}$ and $\beta \geqslant 1$

$$
\hat{f}(\sigma) \leqslant c_{2} f(\beta / \sigma)+c_{3}+c_{5} \hat{f}(\sigma)+c_{6} .
$$

Next we take $\beta \geqslant 1$ such that $\rho(\beta)<\rho(0)$ and $c_{5} \leqslant \frac{1}{2}$. Then for $0<\sigma<\sigma_{0}$

$$
\hat{f}(\boldsymbol{\sigma}) \leqslant 2 c_{2} f(\beta / \sigma)+2\left(c_{3}+c_{6}\right) \text {. }
$$

Then (19), (22) and (27) imply (9) if $f$ is unbounded (i.e. $\hat{f}$ is unbounded), hence (21).

REMARK. If $f$ is bounded, Theorem 1 can be applied (suppose $\rho$ satisfies (8) with $\alpha=0)$.

Karamata [7] considered the case $\hat{f}(1 / t)=O(g(t))(t \rightarrow \infty)$ with $g \in \mathrm{RO}$.

Definition (SEE [1]). A function $f$ is said to be almost decreasing on $\mathbf{R}^{+}$if there exists a constant $0<m \leqslant 1$ such that

$$
f(x) \geqslant m f(y) \text { for each } y \geqslant x>0 .
$$

TheOREM 3. Suppose $f: \mathbf{R}^{+} \rightarrow \mathbf{R}^{+}$is almost decreasing on $\mathbf{R}^{+}$. Moreover, suppose $g \in \mathrm{RO}$ with $a:=$ index $g \leqslant 0$, where the function $g$ is defined by $g(t)=\hat{f}(1 / t)$ $(t>0)$. Suppose

$$
-\int_{0}^{1} t^{\alpha} \rho^{\prime}(t) d t<\infty \text { for some } \alpha<a .
$$

Then the above assumptions imply (9), hence index $f \leqslant 0$.

Proof. Analogous to the proof of Theorem 1 in de Haan and Stadtmüller [6].

Take $b>0$ such that $\rho(b)<\rho(0)$. Then since $f$ is almost decreasing, for $t>0$,

$$
\hat{f}\left(\frac{1}{t}\right) \geqslant-\int_{0}^{b} \rho^{\prime}(s) f(t s) d s \geqslant+c_{1} f(t b)\{\rho(0)-\rho(b)\}=c_{2} f(t b) .
$$

Conversely, using (30) we find

$$
\begin{aligned}
\hat{f}(\sigma) & =-\int_{0}^{\beta} \rho^{\prime}(x) f\left(\frac{x}{\sigma}\right) d x-\int_{\beta}^{\infty} \rho^{\prime}(x) f\left(\frac{x}{\sigma}\right) d x \\
& \leqslant c_{3} f\left(\frac{\beta}{\sigma}\right) \rho(\beta)-c_{4} \int_{0}^{\beta} \rho^{\prime}(x) \hat{f}\left(\frac{b \sigma}{x}\right) d x,
\end{aligned}
$$

where $\beta>0$ is specified later.

Application of the second part of the Lemma gives for $\sigma \leqslant \sigma_{0}$

$$
-\int_{0}^{\beta} \rho^{\prime}(x) \hat{f}\left(\frac{b \sigma}{x}\right) d x \leqslant-c_{5} \hat{f}(\sigma) \int_{0}^{\beta} \rho^{\prime}(x) x^{\alpha} d x .
$$


Take $0<\beta<b$ such that

$$
\rho(\beta)>0 \text { and }-c_{4} c_{5} \int_{0}^{\beta} \rho^{\prime}(x) x^{\alpha} d x<\frac{1}{2} .
$$

Then (31) and (32) imply

$$
\hat{f}(\sigma) \leqslant 2 c_{3} \rho(\beta) f(\beta / \sigma) \quad\left(\sigma \leqslant \sigma_{0}\right) .
$$

Now (30), (33) and $g \in$ RO imply $\hat{f}(1 / t) \asymp f(t)(t \rightarrow \infty)$.

Next we prove a theorem concerning asymptotically balanced functions.

THEOREM 4. Suppose $U: \mathbf{R}^{+} \rightarrow \mathbf{R}$ and there exist a constant $x>1$ and a positive function $f$ such that

$$
U(t x)-U(t) \asymp f(t) \quad(t \rightarrow \infty)
$$

with $f \in \mathrm{RO}$ locally bounded, $\alpha:=\operatorname{index} f$ and $\beta:=\overline{\operatorname{index}} f$. Suppose $\hat{U}(t)$ exists for $t>0$ and (8) is satisfied. Then the above assumptions imply

$$
\hat{U}(1 / t x)-\hat{U}(1 / t) \asymp f(t) \quad(t \rightarrow \infty) .
$$

The proof of the above theorem is a consequence of Theorem 1 . In order to prove a converse statement we suppose the function $U$ to be nondecreasing.

TheOREM 5. Suppose $U: \mathbf{R}^{+} \rightarrow \mathbf{R}^{+}$is nondecreasing, $U(0+)=0$ and define the function $f$ by

$$
f(t)=t^{-1} \int_{0}^{t} s d U(s)
$$

Suppose for some $x>1$

$$
\hat{U}(1 / t x)-\hat{U}(1 / t) \asymp a(t) \quad(t \rightarrow \infty)
$$

where index $a>-1$. If one of the following assumptions is satisfied:

(a) (16), (17), (18) and

$$
-\int_{1}^{\infty} t^{\alpha} \rho^{\prime}(t) d t<\infty \text { for some } \alpha>\text { index } a,
$$

(b) $f$ is almost decreasing on $\mathbf{R}^{+}$, index $a \leqslant 0$ and

$$
-\int_{0}^{1} t^{\alpha} \rho^{\prime}(t) d t \overline{<\infty} \text { for some } \alpha<\text { index } a,
$$

then

$$
U(t x)-U(t) \asymp a(t) \quad(t \rightarrow \infty)
$$

Proof. Note that

$$
\hat{U}\left(\frac{1}{x}\right)-\frac{1}{x} \int_{0}^{x} \hat{U}\left(\frac{1}{s}\right) d s=-\int_{0}^{\infty} \rho^{\prime}(s) f(s x) d s=\hat{f}\left(\frac{1}{x}\right) \text { for } x>0 .
$$

By de Haan and Stadtmüller [6], (37) is equivalent to

$$
t^{-1} \int_{0}^{t} s d \hat{U}(1 / s) \asymp a(t) \quad(t \rightarrow \infty)
$$

with $a \in \mathrm{RO}$, and

$$
\lim _{t \rightarrow \infty} a(t x) / a(t)>x^{-1} \quad \text { for some } x>1 .
$$

The proof is finished by application of Theorems 2 and 3. 


\section{REFERENCES}

1. S. Aljancić and D. Arandelović, 0-regularly varying functions, Publ. Inst. Math. (Beograd) (N.S.) 22 (1977), 5-22.

2. N. H. Bingham and C. M. Goldie, Extensions of regular variation. I, II, Proc. London Math. Soc. (3) 44 (1982), 473-496; 497-534.

3. N. H. Bingham and J. L. Teugels, Tauberian theorems and regular variation, Nieuw. Arch. Wisk. (3) 27 (1979), 153-186.

4. Mercerian and Tauberian theorems for differences, Math. Z. 170 (1980), 247-262.

5. L. de Haan and S. Resnick, Asymptotically balanced functions and stochastic compactness of sample extremes, Ann. Probab. (2) 12 (1984), 588-608

6. L. De Haan and U. Stadtmüller, Dominated variation and related concepts and Tauberian theorems for Laplace transforms, J. Math. Anal. Appl. (to appear).

7. J. Karamata, Über einen Satz von Vijayaraghavan, Math. Z. 34 (1932), 737-740.

Department of Mathematics, Erasmus University, MerellaAN 236, Capelle, AD IJsSel, NETHERLANDS 